\title{
Interactive Teaching of Digital Electronics in Agricultural Universities in China
}

\author{
https://doi.org/10.3991/ijet.v14i05.8855 \\ Longsheng $\mathrm{Fu}^{(\bowtie)}$ \\ Northwest A\&F University, Yangling, China \\ Ministry of Agriculture, Yangling, China \\ Shaanxi Key Laboratory of Agricultural Information Perception and Intelligent Service, \\ Yangling, China \\ Washington State University, Prosser, USA \\ fulsh@nwafu.edu.cn \\ Xin Zhang \\ Washington State University, Prosser, USA \\ Yaqoob Majeed \\ University of Agriculture, Faisalabad, Pakistan \\ Rui Li \\ Northwest A\&F University, Yangling, China;
}

\begin{abstract}
This article exemplifies the establishment of digital electronic course in Northwest A\&F University, which is a typical agricultural university in China, for students who are majoring in electronic information engineering. Three major existing problems were systematically analyzed and discussed, and three potential and feasible solutions were also provided. This article aims at improving the quality of digital electronic course in agricultural universities in China, which can in turn better serve for agricultural engineering.
\end{abstract}

Keywords-Agricultural universities, digital electronics, simulation software, agricultural characteristics

\section{$1 \quad$ Introduction}

The higher education system in China is different from that in developed countries, where at least one agricultural university has been established in each province to support the development of local agriculture [1]. With the rapid development of information technology, the application of electronic technologies in agriculture is becoming increasingly more widespread and important. In order to cater for this, many agricultural universities in China have already started the electronic information engineering/science/technology related majors [2]. Among all, Digital Electronic (DE) is one of the most important professional basic courses, which covers a wide range of topics either in theoretical or practical digital electronics [3]. On the one hand, DE is 
able to help students to build a basic theory structure and to encourage them to innovate in the field of digital electronic technology. On the other hand, it also establishes a solid foundation for those follow-on professional courses and make them possible to be in-depth investigated in specialized fields [4].

Teaching elementary circuit design can be a challenge because Electrical Engineering (EE) students often do not see the immediate relationship between cause and effect, which can be seen, for example, in mechanical engineering experiments [5]. Traditionally, circuit-design teaching follows a three-step sequence from introductory logic to combinatorial circuits, and a few of lectures later, to sequential circuits. The overall goals of the DE course are to analyze and design digital logic circuits, which build a foundation for preparing a student to take follow-on courses and to develop required skills to solve engineering problems. Main topics include digital systems and codes, logic gates, Boolean algebra, logic simplification, combinational logic circuits, flip-flops and related devices, programmable logic devices, sequential logic circuits, large-scale integrated circuits, and analog-to-digital or digital-to-analog converter.

In addition, DE in agricultural universities are somehow different from that in traditional engineering universities [6]. Most graduate students from the major of electronic information engineering (EIE) in agricultural universities have already had some experiences previously or will work in the agricultural engineering (AE) field in the future. Therefore, it would be better to teach DE by integrating with the practical application in the actual AE application, which would also be helpful for the development of students' future careers.

More importantly, EIE is a practical application focused major, and thus the requirement for the hands-on experiments are relatively high [7]. Therefore, the composition of the laboratory is a key part for this major. DE generally not only requires a wide theoretical knowledge system and practical hands-on operations, but also experiences to build a bridge between two sides. Therefore, the theoretical teaching and hands-on exercise of the course needs proper integration and mutual promotion. However, due to the late start of this course in agricultural universities, there are some problems facing. In order to reveal the nature of the problem in this work area, this article aims at analyzing the most commonly seen problems (three 'not easy') along with potential solutions via exemplifying DE course in the major of EIE in Northwest A\&F University, which is a typical agricultural university in China.

\section{State of the Problems}

\subsection{Not easy to start}

EIE students often start with DE as a beginner for the follow-on courses such as algebra and calculation of learning logic [8]. Considering widely used teaching material in China as an example-Digital Electronics Technology Foundation (Fifth Edition), Tsinghua University Electronics Research Group (eds.), Shi Yan (editor), Higher Education Press. The first chapter of the book introduces digital systems and codes, which mainly includes several commonly used number systems, conversions between 
different number systems, the characteristics of binary arithmetic operations, the complement operation, and a few of common encodings. The second chapter introduces Boolean algebra and logic simplification, which mainly includes three basic operations, basic and common formulas, basic theorems, logic functions and their representation in logic algebra, simplification of logic functions (formula simplification method and Karnaugh map simplification method), and the logic function of irrelevant terms and its simplification. Through some personal communications, students often have high expectations of the improvement of their practical ability for this course. If lecturers only teach theories and formulae at the beginning of the course, it will certainly undermine students' enthusiasms. However, these fundamental theories and formulas must be well mastered before diving into subsequent chapters with considerable applications. In addition, some students are unable to start because they have background difficulties with understanding of some theories, which might let them, lose the confidence and feel that "this course is difficult to learn". Therefore, lecturers are not only required to teach the course content clearly, but also to stimulate the enthusiasm of the students, encouraging more interactions and participations in teaching DE.

\subsection{Not easy to innovate in verification experiments}

DE is not only highly theoretical but also highly practical [9]. DE provides certain level of requirements of hands-on ability for students. For example, in the current limited time for theory teaching, it mainly explains the third chapter of gate circuit, the fourth chapter of combinational logic circuit, the fifth chapter of trigger, the sixth chapter of sequential logic circuit, the tenth chapter of pulse waveform generation and shaping, and the eleventh chapter of number-module and analog-to-digital conversion. The course contents are keep increasing, as a result, lecturers have to occupy a few of experimental courses as supplements to theoretical courses. Therefore, the general arrangement of 16 hours for 8 experiments is far away from sufficiency. Experimental courses are normally arranged after theoretical courses have already completed some contents, which has caused discontinuousness between theories and practices. You will need to demonstrate the experiment first if you want to improve the quality of teaching. However, there are four major problems exist:

- It can take considerable time to prepare the experimental demonstration (e.g. connecting the circuit in an experiment box as shown in Fig. 1)

- It is inconvenient to move a few of important instruments and equipment to the classroom

- The demonstration operation process will occupy some time, and therefore, influence the teaching plan

- The reliability of digital circuit in the experimental box is low, which may work normally when it is ready, but cannot work normally during the demonstration. Therefore, in the actual teaching activity, it is only possible to teach pure theories of DE. In this case, students will only be able to know a few of names of digital 
devices and circuit working principles, lacking of digging into specific tasks and applications.

Due to the increase in the number of students, experimental equipment and devices are often over-used lacking of timely maintenance. Normally, experimental project group was supposed to be a two-person group. However, it resulted in that a few of students are not collaborating with others. In the worst case, a few of students tried to exchange the experimental box with others to cheat.

Furthermore, experiments of DE in China mainly focus on validating principles or theories [10]. Generally, experiments can be completed where the experimental instructions are provided. Although students can learn hands-on skills of assigned instruments through experiments. However, without a process of independent thinking, it will be difficult not only to really improve students' ability to find, analyze, and solve problems, but also to stimulate their desire for innovations. Lastly, because of some other random reasons, the success rate is low: most of the time were used for debugging in hands-on courses. The design and comprehensive experiments are difficult for students also due to that, students with different major background are mixed. Therefore, lecturers usually provide reference circuits that do not really serve for cultivating students' innovative abilities.

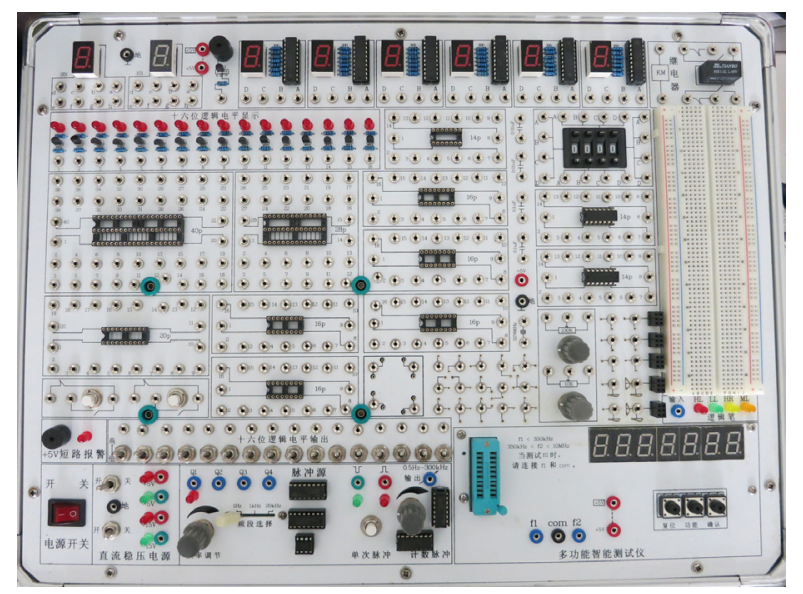

Fig. 1. An experimental box used in Northwest A\&F University for teaching digital electronics (DE) course

\subsection{Not easy to integrate with agricultural characteristics}

The same major has different characteristics in different type of universities [11]. For example, EIE major from University of Electronic Science and Technology focuses on processing and transforming of images, and XiDian University focuses on radar information processing. This major in agricultural universities should emphasize on specific agricultural characteristics. However, since it has been comparatively established for a short period, more agricultural characteristics needs to be correlated. 
Thus, features of our laboratories is not well highlighted, and the experimental content is more about general verification experiments as I mentioned in the previous section. Taking EIE major from Northwest A\&F University as an example, the comprehensive design experiment failed to be properly combined with $\mathrm{AE}$ and many graduate students are even not clear about what is AE. Those students who graduated in recent years can still find a desired job though; they do not have a clear direction of career life, such as what type of industries they will have a chance to be hired with what kind of level of education degree or professional skills. In the "12th Five-Year Plan" of China, the government has incorporated AE into one of the ten key input projects [12]. Therefore, the development of $\mathrm{AE}$ is promising in recent years if $\mathrm{AE}$ and EIE can be properly combined.

Characteristics of the curriculum highly reflects the profession of a student. In order to better develop the $\mathrm{AE}$ education, it is, therefore, required that $\mathrm{DE}$ as a mandatory curriculum. However, how to effectively integrate DE with $\mathrm{AE}$ is still a challenging question that plagues teachers.

\section{Solutions}

\subsection{Combining theoretical teaching with hands-on exercise}

Before the class, lectures need to fully review student's curriculum system, clarify the teaching objectives, and arrange the lecture contents in a reasonable way. Most of students who are majoring in EIE have studied computer basics and $\mathrm{C}$ programming language, and have already had a certain level of understanding of computer languages and programing ability before starting $\mathrm{DE}$. Therefore, when the theory such as binary and digital logic that referring to the basic operation of the computer can be explained as much as possible. For example, the binary arithmetic and the complement operations should be combined with the features of arithmetic operations in the computer language (both of which are binary operations and can only be added by shifting) to explain why binary subtraction is needed. Both multiplication and division need to be converted into addition operations and how they can be implemented by means of an inverted and a complement. Through the combination of theory and practice, students can have more intuitive experiences.

The basic theory of DE is different from that of our daily life [13]. It is often expressed in digital form of 0 and 1 , while our daily life is using 0 to 9 . If students do not master it well, the following contents will be difficult to be understood. Therefore, for this part of the theory, the lecturer has to explain it in a systematical way, such as the simplification of the logic function in chapter two, the formula method and Karnaugh map method are fundamentally important, which need to be clarified in such a detailed way. However, these two methods are difficult to be practically applied in real cases due to the limitations of the number of variables [14]. Instead, the QuinnMcLaskey simplification method (Q-M method) is more practical and can solve practical engineering problems $[15,16]$. However, due to the reform of teaching in recent years, this part of the course has been removed as the result of course hour reduction. 
In order to improve the interests and participations of students, this part has been handed over to the students for self-studying, who will be asked to present with slides on the class. Since students have already learned $\mathrm{C}$ programming language a semester before, they are asked to use $\mathrm{C}$ programming language to implement and demonstrate the Q-M method. In this case, the students was interactive with teachers to learn the basic theory of DE.

\subsection{Introducing simulation software for interactive teaching}

In the previous introductory undergraduate $\mathrm{EE}$ course, students were able to understand digital logic design concepts. Now students will acquire the knowledge of how to initially design a digital logic circuit, which allows them to solve real problems.

Students are requested to develop a combinatorial digital logic circuit that can perform a semi-real-life task. During this process, the truth tables must be derived and then solved into the most efficient Boolean algebra expression by applying the Karnaugh map, the Q-M method, or other algorithms. This expression results are in the final digital circuit diagram, which contain logic gates and connections obtained by the minimization process. Many useful computer programs are available which allow us to achieve various aspects of digital logic design [17-19]. They are ranged from simple simulators, specific teaching tools, to advanced and specialized software. The authors tested a few of these packages aiming to specifically introduce the circuit design teaching, which often perform the functions generally required in undergraduate courses, for example, Karnaugh maps, Q-M method and Espresso minimizations.

Several programs can perform the simulation of a digital circuit, e.g., Proteus [18] and Multisim [19]. However, these commercial software are normally unaffordable by undergraduate students. Therefore, a free software, WinLogiLab [20], an interactive computerized teaching suite developed for the design of combinatorial and sequential logic circuits can be considered. WinLogiLab comprises of a set of tutorials that use the input data provided by student to perform the initial design steps for digital combinatorial and sequential logic circuits. The combinatorial tutorials are designed to show the links between Boolean Algebra and Digital Logic circuits, and follow the initial design steps: from Boolean algebra, truth tables, Exact and the Heuristic minimization techniques, to finally produce the combinatorial circuit. Similarly, the sequential tutorials can design simple State Machine Counters, and can model more complex Finite State Automata.

The structure of WinLogiLab, including its major modules, is depicted in Fig. 2. Its major clusters are the combinatorial and sequential logic modules. In addition, there is a small cluster for the introduction to Numerical Theory. Modules in the combinatorial logic are WinBoolean, BoolTut, and WinEspresso. These modules cover logic gates, Boolean algebra, truth tables, and logic minimization techniques with Karnaugh maps and the Q-M method, and Espresso algorithms. The sequential modules are WinCounter and WinState, which cover state counter design and the design of general-purpose FSMs.

The components of the WinLogiLab tutorial suite can be used in two modes. One mode is a step-by-step, self-paced, set tutorial that guides students through any of its 
tasks and serves as an introduction because it provides specific explanatory information. Alternatively, students can experiment by designing their own circuits in independent self-paced exercises where students use their own inputs. Both modes offer sufficient helps and feedbacks to the student in a self-paced learning mode, in particular, because students can see an immediate relationship in cause and effect through what has happened on the screen, as well as through pop-up feedback comments. This level of understanding would be difficult in conventional Boolean algebra teaching where students do not need to go through all steps until the final circuit is designed. Input can occur at several points throughout the circuit design sequence by using either a manual input or a file saved from an earlier output.

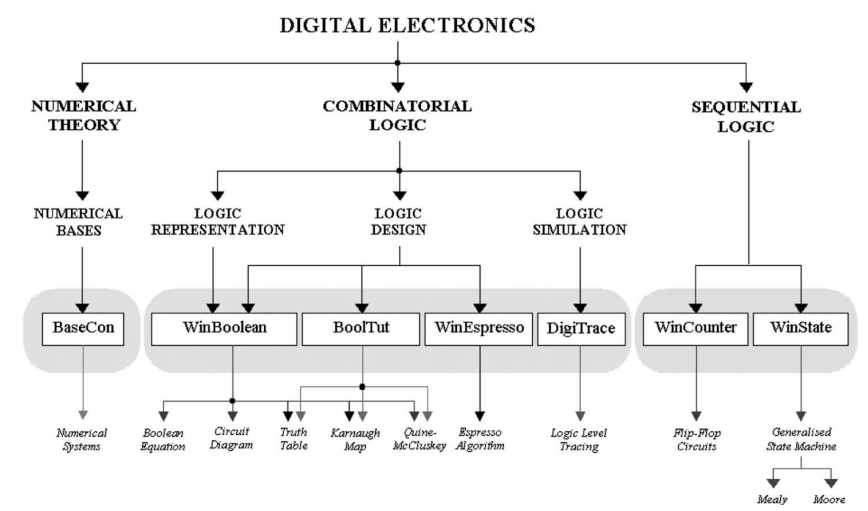

Fig. 2. Structure of WinLogiLab and its relationship with digital electronic (DE) course

Furthermore, self-tests are available to students. In this test, the student is requested to answer a number of computer-generated multiple-choice or short-answer questions on digital logic. For example, the student can be requested to enter the Boolean equation for a given randomly generated circuit diagram. Similarly, other randomly generated questions on circuits, equations, truth tables, or minimization techniques are feasible. A true/false feedback will be given immediately after each answered test question, and a total score of the number of questions that were correctly answered will be given at the end. WinLogiLab is also projector-friendly.

The most important purpose of introducing this free software is to demonstrate the full transition from the numbering system to the testing of the simulated circuit by tracing its functioning to students, where the student can learn by participating. Students are able to learn at their own paces, discovering principles while completing their experiments. Alternatively, they can step through a set of tutorials until they feel confident to use their own inputs.

A student's initial understanding in digital logic design is a tabulated Boolean truth table, which was minimized to a logic circuit schematic or Boolean function. To assess the feasibility of the tutorial suite, we conducted a survey to students of the introductory digital logic subject. The survey was repeated for two consecutive years. According to some participated students, the best feature included that software is a 
good study aid where tutorials make circuits easy to design, to solve Boolean equations, and to understand Karnaugh maps.

In experimental teaching, experiments are divided into basic experiments, design experiments, and comprehensive experiments. Basic experiments (such as integrated logic gate experiments) generally take actual operations, while design experiments and comprehensive experiments are divided into three stages: theoretical design, simulation, and operation. The simulation stage plays an important role. On the one hand, the theoretical design can be modified many times through simulation to verify it's correctness and to further improve its efficiency.

On the other hand, before the actual operation, by simulating the designed circuit, students are able to be familiar with the circuit function, to understand the possible reason of circuit failures and potential solutions in advance. The following is an example of the design experiment "Eight-way Responder". The design requirements of the experiment are as follows: the host presses the "Start" button and the route starts to answer; when the answer is successful, the number of the successful person and the responder is displayed. Until the host presses the reset switch, other people will not respond again. The function is extensible, convenient, and practical. The theoretical design scheme for this is as follows: the input of the answering device is an eight-way answer button and the supporter controls the start and reset of the two buttons. The input of the answer device may have a light emitting diode indicating that the answer is successful, and a digital tube displays the answering person number. A buzzer for sound reminding; because the eight-way switch is turned into a corresponding digital display, and the display decoder receives the BCD code, in this case, the need for 8-3 line encoder, such as 74LS148. It is an integrated device with priority encoding authority. The priority is from high to low according to the access terminal number. In order to enable the digital control to display the corresponding number of each input in a correct way, the reversed code output from 74LS148 must be forwarded. Because the encoder is a combinatorial logic circuit, the signal cannot be held. Once the answer button of a certain route ends, the output code will no longer exist, and the digital cannot be displayed continuously. The latch circuit must be used until the reset signal arrives. For this part, the free software WinLogiLab is inconvenient to describe the whole circuit very well.

Therefore, a more powerful software Proteus [18] should be employed by the lecturer for simulation design to show the students how it works before the experiment. The circuit can be divided into coding section, reset section, display section, and buzzer section, as shown in Fig. 3. Each section of the circuit is designed first, and the problems occurred can be analyzed and solved systematically, and the circuit is gradually improved. 


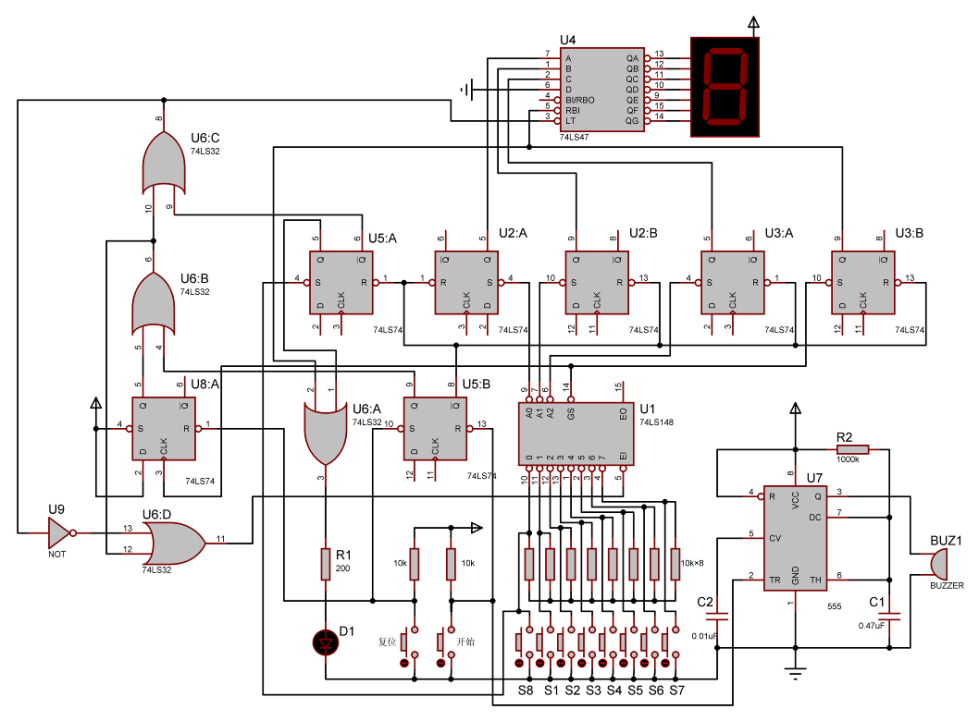

Fig. 3. An example of the design experiment "Eight-way Responder" and its simulation in Proteus

\subsection{Strengthening agricultural characteristics}

Students majoring in EIE can take part in national or provincial electronic competitions for undergraduates after they have studied DE [21,22]. These competitions can improve students' abilities to a certain level. At the same time, they also need some supporting. In most universities, a special electronic innovation laboratory has been established for this purpose. Related courses and majors need to be properly combined with the dominant disciplines of the universities in order to obtain specific characteristics. Taking Northwest A\&F University as an example, AE discipline of university has a long history with many distinguished researches. As early as 1946, it already had a major in agricultural mechanization, and currently has doctoral program and postdoctoral mobile station in AE.

Therefore, we can consider integrating courses and majors into AE, and establishing EIE majors and related courses under the first-level subject of AE. This will not only strengthen the discipline of AE, but also enable EIE and related courses of agricultural colleges to take advantage of their distinguished agricultural background for a better development in the future. In fact, increasingly more AE projects need to be connected with EIE and related courses. For example, topics such as the design of control devices in automatic irrigation of agriculture, seed metering devices in seeding equipment, and the quality nondestructively detection of agricultural products can be considered.

By combining basic professional courses with agriculture, we can highlight the characteristics of the agricultural universities and enable students to understand more about agricultural engineering simultaneously. Therefore, students are able to under- 
stand that this subject and major can make agriculture achieve a leg-up. It will also guide students to establish a sense to serve for agriculture, which will definitely help realizing China's agricultural modernization.

\section{Conclusion}

By exemplifying three existing 'not easy' problems along with three potential solutions in DE course in the major of EIE of Northwest A\&F University in China, this article made a good attempt to comprehensively reveal, analyze, and discuss the nature behind the problem and further by proposing the best solution to address the existing and potential problems. Especially integrating the interactive simulation software (WinLogiLab and Proteus) with the theory lecture can boost the student's interest in this subject, which can lead to uplifting the modernization of China's Agriculture. The Proposed solutions are practical and feasible enough that most of practitioners in this field will benefit from it.

\section{Acknowledgement}

This work was supported by Key Research and Development Program in Shaanxi Province of China (grant number 2018TSCXL-NY-05-04, 2017NY-164), the Education Project of Northwest A\&F University (JY1504045), and the International Scientific and Technological Cooperation Foundation of Northwest A\&F University (grant number A213021505)

\section{References}

[1] J. Huang, R. Hu, S. Rozelle, China's Agricultural Research System and Reforms: Challenges and Implications to the Developing Countries, Asian Journal of Agriculture and Development. 1 (2004) 1-17.

[2] Y. Chen, B. Liu, K. Qi, K. Wang, Computer Supported Control Engineering Education Reform in Agriculture Engineering Field Based on Intelligent Agriculture (IA) New Concept, IERI Procedia. 2 (2012) 603-608. https://doi.org/10.1016/j.ieri.2012.06.141

[3] J. Macias-Guarasa, J.M. Montero, R. San-Segundo, A. Araujo, O. Nieto-Taladriz, A Project-Based Learning Approach to Design Electronic Systems Curricula, IEEE Transactions on Education. 49 (2006) 389-397. https://doi.org/10.1109/TE.2006.879784

[4] N. Dahnoun, Teaching electronics to first-year non-electrical engineering students, International Journal of Electrical Engineering Education. 54 (2017) 178-186. https://doi.org/10.1177/0020720917692345

[5] O.B. Adamo, P. Guturu, M.R. Varanasi, An innovative method of teaching digital system design in an undergraduate electrical and computer engineering curriculum, in: 2009 IEEE Int. Conf. Microelectron. Syst. Educ., IEEE, 2009: pp. 25-28. https://doi.org/10.110 9/MSE.2009.5270837 
[6] M.A. Soderstrand, The new electrical and computer engineering curricula at University of California-Davis, IEEE Transactions on Education. 37 (1994) 136-146. https://doi.org/10.1109/13.284984

[7] L. Li, Project-based learning in electronic technology: a case study, European Journal of Engineering Education. 40 (2015) 499-505. https://doi.org/10.1080/03043797.2014.987650

[8] M. Mantri, Archana Dutt, Sunil Gupta, J. P. Chitkara, Using PBL to Deliver Course in Digital Electronics., Advances in Engineering Education. 1 (2009).

[9] F.J. Azcondo, A. de Castro, C. Branas, Course on Digital Electronics Oriented to Describing Systems in VHDL, IEEE Transactions on Industrial Electronics. 57 (2010) 3308-3316. https://doi.org/10.1109/TIE.2010.2040557

[10] N. ZHENG, W. ZHANG, Q. BAI, Reform of Experimental Teaching of Electronic Technology under the Background of Engineering Education, in: 2017 3rd Int. Conf. Manag. Sci. Innov. Educ., 2017: pp. 378-382.

[11] J.G. Thursby, R. Jensen, M.C. Thursby, Objectives, Characteristics and Outcomes of University Licensing: A Survey of Major U.S. Universities, The Journal of Technology Transfer. 26 (2001) 59-72. https://doi.org/10.1023/A:1007884111883

[12] W. Pang, J.A. Plucker, Recent Transformations in China's Economic, Social, and Education Policies for Promoting Innovation and Creativity, The Journal of Creative Behavior. 46 (2012) 247-273. https://doi.org/10.1002/jocb.17

[13] S. Malik, L. Zhang, Boolean satisfiability from theoretical hardness to practical success, Communications of the ACM. 52 (2009) 76. https://doi.org/10.1145/1536616.1536637

[14] M.E. Holder, A Modified Karnaugh Map Technique, IEEE Transactions on Education. 48 (2005) 206-207. https://doi.org/10.1109/TE.2004.832879

[15] N.N. Biswas, Minimization of Boolean Functions, IEEE Transactions on Computers. C-20 (1971) 925-929. https://doi.org/10.1109/T-C.1971.223373

[16] Z. Gan, T. Shang, G. Shi, C. Chen, Automatic Synthesis of Combinational Logic Circuit with Gene Expression-Based Clonal Selection Algorithm, in: 2008 Fourth Int. Conf. Nat. Comput., IEEE, 2008: pp. 278-282. https://doi.org/10.1109/ICNC.2008.338

[17] A. Hacker, A Computer-Based Interactive Teaching Software for the Tracing of Logic Levels in a Digital Circuit, Global Journal of Engineering Education. 6 (2002) 85-90.

[18] EDA for Education - Read how Proteus is used to teach electronics - Labcenter Electronics, (n.d.). https://www.labcenter.com/education/ (accessed April 20, 2018).

[19] NI Multisim: Powerful Circuit Design and Teaching Software - National Instruments, (n.d.). http://www.ni.com/multisim/ (accessed April 20, 2018).

[20] WinLogiLab - HakaSoft Software, (n.d.). http://www.hakasoft.com.au/winlogilab (accessed April 20, 2018).

[21] J. Peng, H. Xie, Y. Feng, L. Fu, S. Sun, Y. Cui. Simulation study of vibratory harvesting of Chinese winter jujube (Zizyphus jujuba Mill. cv. Dongzao). Computers and Electronics in Agriculture. 143 (2017) 57-65. https://doi.org/10.1016/j.compag.2017.09.036

[22] T. Liu, H. Sun, Analysis of Information Literacy Education Strategies for College Students Majoring in Science and Engineering, Modern Applied Science. 5 (2011) 227. https://doi.org/10.5539/mas.v5n5p227

\section{$7 \quad$ Authors}

Longsheng Fu (corresponding author) is an associate professor in College of Mechanical and Electronic Engineering, Northwest A\&F University, Yangling 712100, 
China, and an adjunct faculty in Center for Precision and Automated Agricultural Systems, Washington State University, Prosser, 99350, USA.

Xin Zhang is a Ph.D. student in Center for Precision and Automated Agricultural Systems, Washington State University, Prosser, 99350, USA.

Yaqoob Majeed is a lecturer in Faculty of Agricultural Engineering and Technology, University of Agriculture, Faisalabad 38000, Pakistan.

Rui Li is a lecturer in College of Mechanical and Electronic Engineering, Northwest A\&F University, Yangling 712100, China.

Article submitted 2018-05-15. Resubmitted 2018-09-27. Final acceptance 2018-09-27. Final version published as submitted by the authors. 